POS $\quad \begin{aligned} & \text { PROCEEDINGS } \\ & \text { OF SCIENCE }\end{aligned}$

\title{
ALICE TPC - design and performance
}

\author{
A. Matyja*1, M. Kowalski ${ }^{1}$, D. Roehrich ${ }^{2}$, D. T. Larsen ${ }^{2}$, D. Fehlker ${ }^{2}$, H. Helstrup ${ }^{3}$,
} B. Sitar ${ }^{4}$, M. Pikna ${ }^{4}$, M. Siska ${ }^{4}$, R. Janik ${ }^{4}$, P. Strmen ${ }^{4}$, I. Szarka ${ }^{4}$, L. Musa ${ }^{5}$, C. Lippmann ${ }^{5}$, M. Mager ${ }^{5}$, A. U. Rehman ${ }^{5}$, S. Rossegger ${ }^{5}$, B. S. Nielsen ${ }^{6}$, C. Soegaard ${ }^{6}$, H. Oeschler ${ }^{7}$, A. Kalweit ${ }^{7}$, H. Appelshaeuser ${ }^{8}$, R. Renfordt ${ }^{8}$, P. Braun-Munzinger ${ }^{9}$, H.-R. Schmidt ${ }^{9}$, D. Vranic $^{9}$, C. Garabatos ${ }^{9}$, U. Frankenfeld ${ }^{9}$, M. Ivanov ${ }^{9}$, Y. Foka ${ }^{9}$, J. Stachel ${ }^{10}$, P. Glassel ${ }^{10}$, J. Wiechula ${ }^{10}$, H. K. Soltveit ${ }^{10}$, H.-A. Gustafsson ${ }^{11}$, P. Christiansen ${ }^{11}$, A. Oskarsson ${ }^{11}$, P. Gros ${ }^{11}$, A. F. Dobrin ${ }^{11}$

${ }^{1}$ Institute of Nuclear Physics PAN, ul. Radzikowskiego 152, 31-342 Kraków, Poland

${ }^{2}$ Department of Physics and Technology, University of Bergen, Bergen, Norway

${ }^{3}$ Faculty of Engineering, Bergen University College, Bergen, Norway

${ }^{4}$ Faculty of Mathematics, Physics and Informatics, Comenius University, Bratislava, Slovakia

${ }^{5}$ European Organization for Nuclear Research (CERN), Geneva

${ }^{6}$ Niels Bohr Institute, University of Copenhagen, Copenhagen, Denmark

${ }^{7}$ Institut für Kernphysik, Technische Universität Darmstadt, Darmstadt, Germany

${ }^{8}$ Institut für Kernphysik, Johann-Wolfgang-Goethe Universität Frankfurt, Frankfurt, Germany

${ }^{9}$ Gesellschaft für Schwerionenforschung mbH (GSI), Darmstadt, Germany

${ }^{10}$ Physikalisches Institut, Ruprecht-Katls-Universität Heidelberg, Heidelberg, Germany

${ }^{11}$ Division of Experimental High Energy Physics, University of Lund, Lund, Sweden

E-mail: adam.matyjalifj.edu.pl, Marek.Kowalski@cern.ch

Dieter.Rohrich@fi.uib.no Dag.Larsenecern.ch sitarefmph.uniba.sk

Dominik.Fehlker@uib.no Alexander.Philipp.Kalweitecern.ch

Miroslav.Pikna@cern.ch, Martin.Siska@cern.ch janikefmph.uniba.sk

Peter.Strmen@cern.ch Imrich.Szarka@cern.ch, Luciano.Musalcern.ch

Christian.Lippmannecern.ch Chilo.Garabatos.Cuadradodcern.ch

Magnus.Mager@cern.ch Attiq.Ur.Rehman@cern.ch H.R.Schmidt@gsi.de

Stefan.Rossegger@cern.ch Borge.Nielsendcern.ch, soegaardenbi.dk

h.oeschleregsi.de, appels@ikf.uni-frankfurt.de.

Marian.Ivanovecern.ch, Rainer.Renfordtecern.ch,

B.Braun-MunzingereGSI.De Yiota.Foka@eern.ch U.FrankenfeldeGSI.De

Haavard.Helstrup@cern.ch Danilo.Vranicecern.ch,

stachelephysi.uni-heidelberg.de glasselephysi.uni-heidelberg.de

ALICE is the dedicated heavy ion experiment at the LHC. The detector is optimised to register heavy ion collisions, up to $\mathrm{Pb}-\mathrm{Pb}$, as well as p-p. The main tracking device of the ALICE experiment is the Time Projection Chamber - TPC. We will present the design of the detector and its performance. The current status of the detector and the capabilities to underlying physics, based on the cosmic rays tests, will be shown as well.

The 2009 Europhysics Conference on High Energy Physics,

July $16-222009$

Krakow, Poland

*Speaker. 


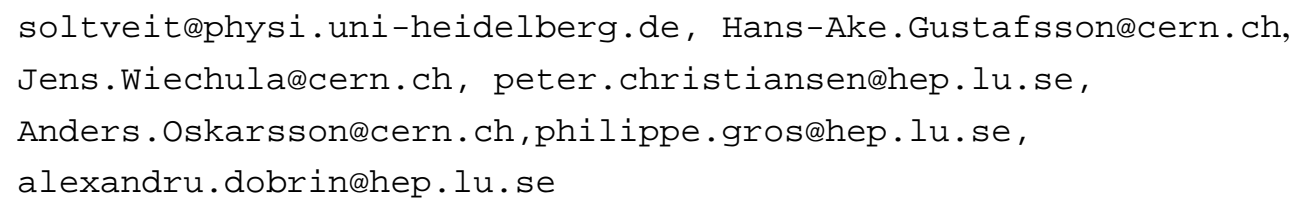

\section{Design}

The ALICE experiment [1] is the dedicated heavy-ion experiment at LHC. Its main tracking device is the Time Projection Chamber (TPC) [2]. The TPC has been designed to cope with the highest, up to 8000 , charged particle multiplicities per rapidity unit in the central region. The TPC is a $5 \mathrm{~m}$ diameter cylinder with a length of $5 \mathrm{~m}$. The central electrode has a voltage of $100 \mathrm{kV}$ giving rise to a drift field of $400 \mathrm{~V} / \mathrm{cm}$. The drift volume $\left(90 \mathrm{~m}^{3}\right)$ is divided into two readout parts. The readout chambers (ROC), installed at the two endplates of the cylinder are divided into 18 sectors. Each sector consists of an inner and an outer chamber (IROC and OROC, respectively). The operation is based on the multiwire proportional chamber technique with pad readout. There are 557568 readout pads with three different sizes allocated on 72 ROCs.

The cold gas mixture $\mathrm{Ne}-\mathrm{CO}_{2}-\mathrm{N}_{2}(85.7-9.5-4.8 \%)$ [3] requires temperature stability and homogeneity less than $0.1 \mathrm{~K}$ [4] within the whole TPC volume. This is required to achieve the non-saturated drift velocity $(2.7 \mathrm{~cm} / \mu \mathrm{s})$.

These requirements were achieved. The TPC works stably without trips. The drift voltage system provides a constant electric field. The recirculating gas system removes $\mathrm{O}_{2}$ and $\mathrm{H}_{2} \mathrm{O}$ contamination on the level of $\sim 1 \mathrm{ppm}$ (design was $<5 \mathrm{ppm}$ ). Thanks to nearly 60 adjustable cooling circuits and around 500 temperature sensors the cooling system provides temperature stability given by specifications. The TPC is fully controlled by the Detector Control System which ensure a safe and correct operation.

\section{Calibration}

The main goal of the calibration is to provide the information needed for the reconstruction software. Fig. 11 shows the measured noise distribution both for all chambers and separately for the different chambers. The achieved noise for the whole TPC is about $700 e^{-}$well below the designed mean noise level of $1 \mathrm{ADC}$ count $\left(1000 e^{-}\right)$.

The efficient zero suppression (ZS) must be performed on the level of the Front-End Electronics to handle the huge amount of data. The ZS decreases the data volume from $\sim 700 \mathrm{MB} /$ event to less than $70 \mathrm{kB} /$ event for empty events. It allows for data rates up to $1 \mathrm{kHz}$ for $\mathrm{p}-\mathrm{p}$ and a few 100 $\mathrm{Hz}$ for $\mathrm{Pb}-\mathrm{Pb}$ collisions at $\mathrm{LHC}$ energies.

Inhomogeneities in the drift field together with the magnetic field give rise to an $E \times B$ effect. The calibration of this effect allows precise reconstruction of particle tracks. A laser system is used for that purpose. For each of 336 laser beams and several magnetic field settings the deviation of the electron drift $\Delta r \phi$ from the ideal path is measured (Fig. ש). The $E \times B$ effect is as large as $7 \mathrm{~mm}$ for the longest drift distance and the nominal field. This corresponds to the designed precison of the correction of less than $1 \%$. 


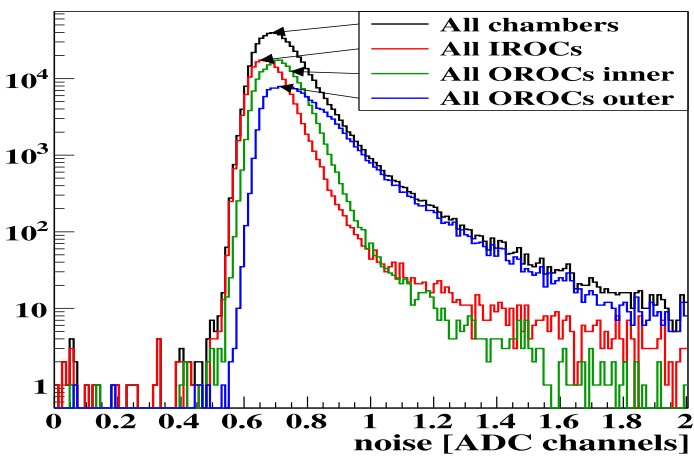

Figure 1: The noise distribution within the TPC, both for all chambers and separately for the different chambers.

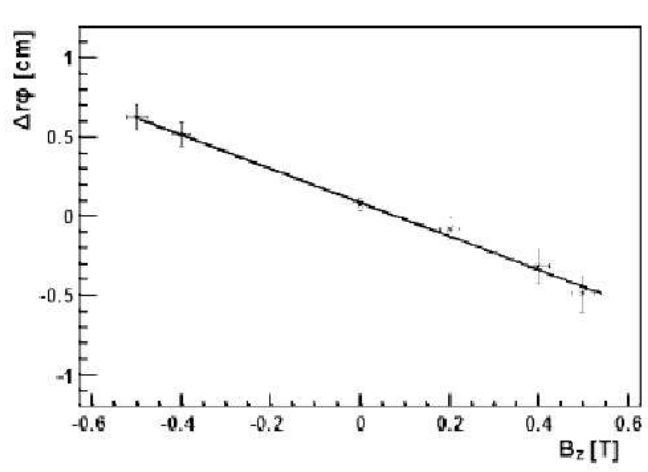

Figure 2: A deviation of the electron drift from the ideal path caused by the $E \times B$ effect as a function of the magnetic field.

The drift velocity is a function of the electric and the magnetic field, the atmospheric pressure and the gas temperature and composition. Its measurement is crucial for track matching with other detectors. It is required to monitor the drift velocity about once per hour to achieve the relative resolution on the level of $6 \times 10^{-5}$.

To obtain an information of the detector response an absolute gain calibration of each pad is needed. The radioactive ${ }_{36}^{83} \mathrm{Kr}$ is injected into the TPC gas system. The characteristic decay spectrum is accumulated for each channel then the position of the main peak (corresponding to energy of $41.6 \mathrm{keV}$ ) is fitted with a Gaussian function. By comparison of the spectra in various TPC parts calibration constants are obtained. The error on the mean obtained from the fit is of the order $0.2 \%$ on the single pad level (required is $1.5 \%$ ). The calibrated spectrum of $\mathrm{Kr}$ decays for all OROCs is shown in Fig. 3. The resolution of the main peak is $4.0 \%$ for IROCs and $4.3 \%$ for OROCs.

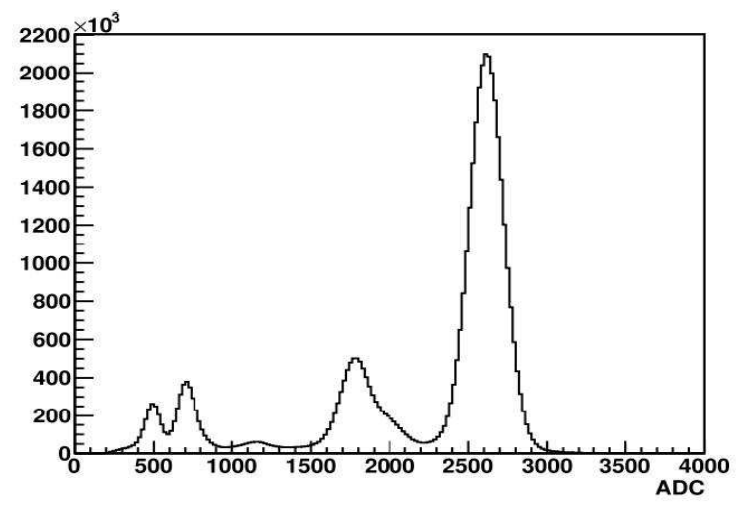

Figure 3: The calibrated Kr spectrum of all OROCs.

\section{Performance}

Over 5 million cosmic muon tracks are collected to determine the transverse momentum $\left(p_{T}\right)$ resolution. The tracks are treated independently in the two halves of the TPC. The resolution 
obtained by comparison of the transverse momentum of two reconstructed track parts at vertex is shown in Fig. 4 as a function of $p_{T}$. The measured resolution at $10 \mathrm{GeV}(6.5 \%)$ is larger than the designed value (4.5\%). It will be improved soon after the complete calibration.

The space point resolution in the $r \phi$ direction, $\sigma_{y}$, depends on the drift length and the inclination angle $\phi$. The results are shown in Fig. 5. The values range from $300-800 \mu \mathrm{m}$ for small inclination angles (high momentum tracks). These values correspond to the expected from simulations.

The measurement of the $d E / d x$ resolution was done using about $7 \times 10^{6}$ cosmic tracks. The measured resolution is less than $5.7 \%$ which is very close to the design value $(5.5 \%)$. The energy loss measurement allows for particle identification up to $50 \mathrm{GeV} / c$.

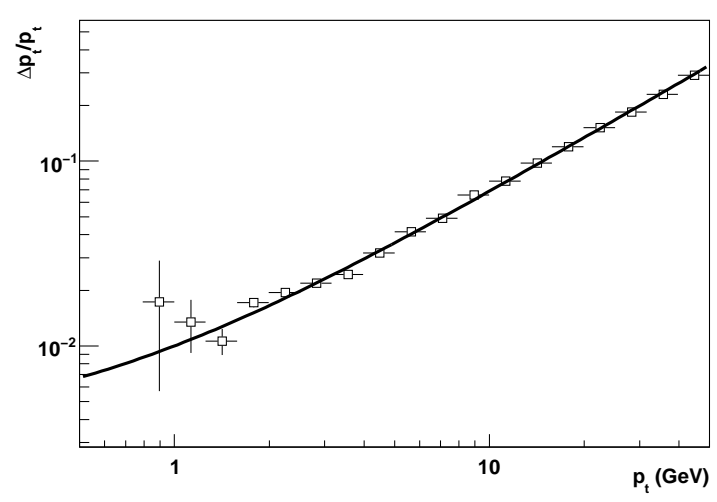

Figure 4: Relative $p_{T}$ resolution.

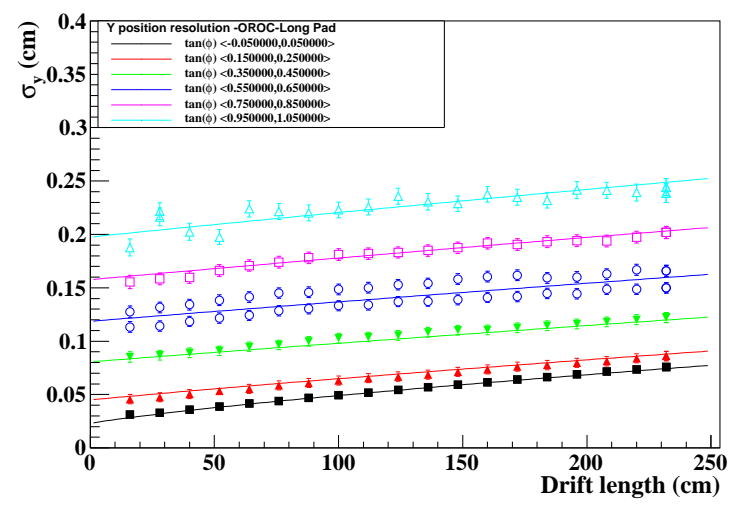

Figure 5: The space point resolution as a function of a drift length for several ranges of inclination angle.

\section{Conclusions}

The commissioning phase is completed. The TPC is working stably. The calibration is done, however there are ongoing works on improvements. The TPC achieved very good performance close to specifications. The detector has been ready for the first collisions since summer 2008.

\section{References}

[1] K. Aamodt et al. (ALICE Collaboration), The ALICE experiment at the CERN LHC, JINST 3 (2008) S08002.

[2] ALICE Collaboration, ALICE time projection chamber : Technical Design Report, ALICE TDR 7, CERN/LHCC 2000-001 (2000).

[3] C. Garabatos (ALICE Collaboration), The ALICE TPC, Nucl. Instrum. Meth. A535 (2004) 197 [doi:10.1016/j.nima.2004.07.127].

[4] J. Wiechula et al., High-precision measurement of the electron drift velocity in Ne CO-2, Nucl. Instrum. Meth. A548 (2005) 582 [doi:10.1016/j.nima.2005.05.031] 